\title{
Breve estudio bibliométrico sobre economía solidaria
}

Brief bibliometric study on solidarity economy

\author{
Breve estudo bibliométrico sobre economia solidária
}

\author{
Ehyder Mario Barbosa Pérez ${ }^{1}$ \\ Henry Vargas Pacheco ${ }^{2}$ \\ Dustin Tahisin Gómez Rodríguez ${ }^{3}$
}

Recibido: 5 de octubre de 2019

Aprobado: 30 de agosto de 2020

Publicado: 30 de septiembre de 2020

Cómo citar este artículo:

Barbosa Pérez, E.M., Vargas Pacheco, H. y Gómez Rodríguez, D.T. (2020). Breve estudio bibliométrico sobre economía solidaria. Cooperativismo \& Desarrollo, 28(118), 1-20. doi: https://doi.org/10.16925/2382-4220.2020.03.05

Artículo de investigación. https://doi.org/10.16925/2382-4220.2020.03.05

1 Director Técnico de Desarrollo de la Unidad Especial Administrativa de Organizaciones Solidarias.

Correo electrónico: ehyder.barbosa@orgsolidarias.gov.co

ORCID: https://orcid.org/0000-0001-5105-4496

2 Estudiante de último semestre de Sistemas de Información, Bibliotecología y Archivística de la Universidad de la Salle.

Correo electrónico: hvargas04@unisalle.edu.co

ORCID: https://orcid.org/0000-0001-7580-2700

3 Estudiante de tercer año del Doctorado en Agrociencias de la Universidad de la Salle. Docente investigador de la Uniagustiniana.

Correo electrónico: dustin_gomez@uniagustinaina.edu.co

ORCID: https://orcid.org/0000-0001-5359-2300 


\section{Resumen}

En este estudio se realizó un análisis bibliométrico de la producción científica (282 artículos) de la categoría economía solidaria en el periodo 2010-2019 en la base de datos Web of Science. Se identificó un aumento en el número de publicaciones sobre economía solidaria hasta 2017. La gran mayoría de los documentos fueron artículos científicos (76\%), seguidos de capítulos de libro. Los autores con mayor número de publicaciones fueron S. Calvo y A. Morales, mientras que las organizaciones con mayor producción fueron la Universidad Cooperativa de Colombia y la Bournemouth University. El país con mayor producción fue Brasil, y Colombia ocupó el séptimo puesto, lo cual ratifica la necesidad de ampliar y enriquecer el acervo científico del país frente a la economía solidaria.

Palabras claves: asociatividad, bibliometría, desarrollo económico, economía solidaria, análisis bibliométrico.

\section{Descriptores:}

B55 Economía social

010 Desarrollo económico

C10 Métodos y metodología econométricos y estadísticos

\section{Abstract}

In this study, we carried out a bibliometric analysis of scientific production (282 articles) of the solidarity economy category in the 2010-2019 in the Web of Science database. An increase in the number of publications on Solidarity Economy until 2017 was identified. Most documents were scientific (76\%), followed by book chapters. The authors with the highest number of publications were S. Calvo and A. Morales. In contrast, the organizations with the highest production were the Cooperative University of Colombia and the University of Bournemouth. The country with the highest production was Brazil, and Colombia is in seventh place, which confirms the need to expand and enrich its scientific heritage vis-à-vis the solidarity economy.

Keywords: associativity, bibliometric, economic development, solidarity economy, bibliometric analysis.

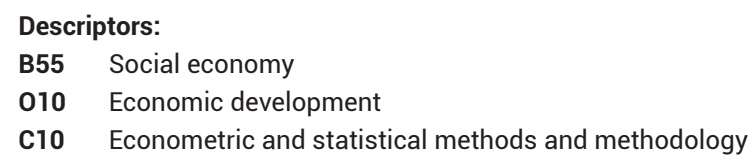

\section{Resumo}

Uma análise bibliométrica da produção científica (total de 282 artigos) da categoria economia solidária foi realizada na janela 2010-2019 na base de dados Web of Science. Foi identificado um aumento no número de publicações sobre Economia Solidária até 2017. A grande maioria dos documentos era científica (76\%), seguida de capítulos de livros. Os autores com maior número de publicações foram S. Calvo e A. Morales, enquanto as organizações com maior produção foram a Universidade Cooperativa da Colômbia e a Universidade de Bournemouth. O país com maior produção foi o Brasil, e a Colômbia ocupa o sétimo lugar, o que confirma a necessidade de expandir e enriquecer o patrimônio científico do país em relação à economia solidária.

Palavras-chave: associatividade, bibliometria, desenvolvimento econômico, economia solidária, análise bibliométrica.

\section{Descritores:}

Economia social B55

010 Desenvolvimento econômico

C10 Métodos e metodologia econométricos e estatísticos 


\section{Introducción}

La Unidad Administrativa Especial de Organizaciones Solidarias (UAEOS) es una entidad pública de orden nacional y adscrita al Ministerio de Trabajo de Colombia. Su misión es promover, fortalecer y desarrollar socioempresarialmente las organizaciones solidarias para generar desarrollo en los sectores y en las regiones del país, con una institucionalidad del sector fortalecida y transversal. Además, busca el posicionamiento del sector solidario en la contribución de logros de paz y equidad en Colombia. "En otras palabras, la estrategia es el servicio para todos sus asociados, la rentabilidad del capital porque este es un medio de trabajo y no la finalidad exclusiva de la acción empresarial" (Monsalve, 2018, p. 45). En el marco de las competencias legales asignadas, debe diseñar, adoptar, dirigir, coordinar y ejecutar los programas y proyectos para la promoción, la planificación, la protección, el fortalecimiento y el desarrollo de las organizaciones solidarias en Colombia (UAEOS, 2019a).

El concepto de economía solidaria está vinculado a los modelos de integración económica solidaria que abarcan expresiones de asociatividad entre organizaciones y comunidades que permiten articular procesos sociales y económicos de producción, consumo, distribución y ahorro (UAEOS, 2019b; Departamento Administrativo Nacional de la Economía Solidaria, 2005). En Colombia, se han presentado desarrollos teórico-conceptuales gracias a experiencias diversas y existe un marco normativo de la economía solidaria presente en la Ley 454 de 1998, que la define como:

Sistema socioeconómico, cultural y ambiental conformado por el conjunto de fuerzas sociales organizadas en formas asociativas identificadas por prácticas autogestionarias solidarias, democráticas y humanistas, sin ánimo de lucro para el desarrollo integral del ser humano como sujeto, actor y fin de la economía.

El marco normativo colombiano busca establecer una manera diversa y plural de identificar la expresión de economía solidaria como identidad basada en una lógica económica con relaciones de solidaridad, reciprocidad y cooperación para ampliar la visión empresarial. La comunidad establece factores económicos en los cuales el trabajo autogestionado condensa factores que se afianzan en las empresas de economía solidaria.

Se puede decir que existe un consenso teórico frente a la economía solidaria que establece un planteamiento de la integración de los eslabones de la economía a partir de principios solidarios como cambio epistemológico de la forma de hacer y pensar la economía (Guerra, 2014 ; Razeto, 1992). En este desarrollo teórico, la noción 
de integración económica remite al territorio, a la comunidad y al relacionamiento entre emprendimientos solidarios, con el propósito de potencializar las capacidades locales y así satisfacer las necesidades para lograr cambios paradigmáticos en la forma de producir y definir las preferencias de consumo (Rúa Castañeda et al., 2016). Así, la economía solidaria se relaciona con varias corrientes teóricas, contextos socioeconómicos y corporativos, y prácticas empresariales y asociativas. Al respecto, Laville (2004, 2007 ; Laville et al., 2006) indica que la economía solidaria

[...] no se limita a un sinnúmero de actividades económicas con finalidad social, sino que se origina en un movimiento ideológico de la economía y la política. En concordancia con estos autores, emerge como una manera de democratizar la economía a partir de responsabilidades cívicas. (Pérez y Uribe, 2016, p. 538 y 539)

El presente artículo nace de la necesidad de entender lo mencionado, así como de identificar y ampliar las tendencias y líneas argumentativas de la economía solidaria, en virtud de un proyecto interinstitucional entre la Universitaria Agustiniana y la UAEOS titulado "Análisis, desarrollo, fomento y gestión del talento humano en el municipio de Aracataca, Magdalena desde la economía solidaria (2020-2022)". Su primera fase efectúa un análisis documental de las categorías economía solidaria u organizaciones solidarias (2020). Para ello, se ejecuta un análisis bibliométrico, con el cual se podrán identificar las tendencias, los autores, las revistas y los países donde se origina la producción investigadora de esta categoría o enfoque.

Se ha utilizado una de las bases de datos más reconocida internacionalmente: Web of Science (wos), ya que reúne las publicaciones con mayor nivel de impacto y, por tanto, se considera de mayor calidad académica (Bensman y Leydesdorff, 2009; Aguilera Padro et al., 2020). Para terminar, el presente artículo, es un análisis bibliométrico de la categoría economía solidaria, para el cual se toma como ventana de observación el periodo comprendido entre 2010 y 2019, con el objetivo de identificar las tendencias bibliométricas de esta categoría.

\section{Marco teórico}

Desde una mirada amplia de la historia y teniendo en cuenta a Bastidas (2015), se puede afirmar que la economía solidaria nace como una contraargumentación frente a los nefastos desequilibrios sociales ocasionados por la revolución industrial en el Reino Unido y que después se expandió por el mundo en el siglo XIX. En efecto, las 
grandes diferencias en la distribución de los ingresos ocasionadas por la acumulación capitalista favorecieron la constitución de organizaciones socioeconómicas de autodefensa con sustentos asociativos, cooperativos y mutuales. "La noción de servicio social organizado por la acción de asistencia mutua, con el único objeto del bien común, genera un universo económico diferenciado del contexto de la economía de mercado que opera en función de la ganancia óptima" (Vainstok, 1985, p. 24, citado por Cueto Fuentes et al., 2018, p. 26).

La economía solidaria no es un producto del sistema socialista, dada la crítica al capitalismo en al anterior párrafo. Lo que sí se puede afirmar es que la economía solidaria es una alternativa que busca el equilibrio entre la libertad del individuo y el bien común desde la óptica de la equidad, la proporcionalidad y la justicia.

Por ende, la economía solidaria, es otra forma de ver la relación entre economía, hombre y su contexto, en donde fundamenta sus acciones en prácticas de lealtad con la cultura, la humanidad, el territorio, en busca de un equilibrio entre el ser, el tener y el vivir. (Dávila et al., 2018, p. 100)

En consecuencia, la economía solidaria no puede ser equiparada con el capitalismo ni con el socialismo, ya que no busca asimilarse como un modo de producción, sino que es una estructura productiva entre los factores económicos (Álvarez Rodríguez y López-Santamaría, 2018). Por consiguiente, no se sustenta en términos de poder, es decir, de una jerarquización, ya que no representa relaciones de dominación o de diferenciaron de clases sociales (Vélez Tamayo, 2014, pp. 19-20). De igual modo, no es una teoría consolidada, sino más bien una respuesta ante las injusticias de los sistemas socialista y capitalista (Razeto, 2014, 1997, 1992).

La bibliometría es la aplicación de herramientas estadísticas para el análisis de las comunicaciones escritas de calidad científica, así como de los autores que generan tal producción (Pérez, 2002). De igual modo, la bibliometría hace parte de la sociología de la ciencia y desde ahí indaga datos con los cuales se pueda identificar la conducta de los autores. Por lo anterior, en la bibliometría se puede calcular una serie de indicadores de acuerdo con la diversidad de los documentos (Escorcia Otálora, 2008; Gepp et al., 2018).

A la bibliometría también se le puede denominar como un cúmulo de técnicas cuantitativas que se aplican en la identificación y el análisis de documentos, productos como consumidores y también una herramienta que observa el estado de la tecnología y de la ciencia por medio de la producción global de la literatura científica. Desde esta óptica, la comunidad internacional, en cabeza de la Organización para 
la Cooperación y el Desarrollo Económico (OCDE) y la Organización de las Naciones Unidas para la Educación (Unesco), ha desarrollado manuales que recogen metodologías para la construcción de indicadores en las diferentes ciencias, por ejemplo, el Manual de Frascati, el de Oslo y el de Camberra. Sin embargo, dado lo multidisciplinario de los estudios bibliométricos, estos se nutren también de informática, estadística, entre otros (Solano López et al., 2009; Nunes Alves et al., 2016).

\section{Metodología}

El presente artículo de investigación tiene una metodología de corte cuantitativo y utiliza un análisis bibliométrico de la categoría economía solidaria. En efecto, la medición, la producción y la difusión de la ciencia han tomado relevancia últimamente, en virtud de los sistemas de información que contribuyen al análisis de la producción científica (Castro Jiménez et al., 2017; Gómez Rodríguez et al., 2017a, 2017b, 2017c).

Tabla 1. Inteligencia tecnológica - registro de búsqueda de información

\begin{tabular}{|c|c|c|c|}
\hline \multirow[b]{3}{*}{$\begin{array}{l}\text { BASE DE } \\
\text { DATOS }\end{array}$} & \multicolumn{3}{|c|}{ INTELIGENCIA TECNOLÓGICA - REGISTRO BÚSQUEDA DE INFORMACIÓN } \\
\hline & \multicolumn{3}{|c|}{ Proyecto: Estudio Bibliométrico sobre economía solidaria } \\
\hline & Ecuaciones de Búsqueda & $\begin{array}{c}\text { No. } \\
\text { REGISTROS }\end{array}$ & COMENTARIOS \\
\hline \multirow{5}{*}{ Wos } & TEMA: (Solidarity Economy") & & \\
\hline & $\begin{array}{l}\text { Índices=BKCI-S, ESCI, SSCI, BKCI-SSH, SCI-EXPANDED, } \\
\text { IC, A\&HCI, CPCI-SSH, CPCI-S, CCR-EXPANDED Período de } \\
\text { tiempo=Todos los años }\end{array}$ & 282 & \\
\hline & TEMA: (Solidarity Economy") & \multirow{3}{*}{276} & \multirow{3}{*}{$\begin{array}{l}\text { Filtrado por años } \\
\text { desde } 2010 \text { a } 2019\end{array}$} \\
\hline & $\begin{array}{l}\text { Refinado por: AÑOS DE PUBLICACIÓN: ( } 2019 \text { OR } 2015 \\
\text { OR } 2011 \text { OR } 2018 \text { OR } 2014 \text { OR } 2010 \text { OR } 2017 \text { OR } 2013 \text { OR } \\
2016 \text { OR 2012) }\end{array}$ & & \\
\hline & $\begin{array}{l}\text { Índices=SCI-EXPANDED, SSCI, A\&HCI, CPCI-S, CPCI-SSH, } \\
\text { BKCl-S, BKCI-SSH, ESCI, CCR-EXPANDED, IC Período de } \\
\text { tiempo=Todos los años }\end{array}$ & & \\
\hline
\end{tabular}

Fuente: elaboración propia, a partir de datos de la base datos wos.

Teniendo en cuenta la tabla 1, se realizan unas ecuaciones de búsqueda para recuperar la información de la base de datos escogida. Por este motivo, se inicia la fusión con el programa para el posterior análisis de datos como la minería de textos en Vantage Point, como el Dataset de la base, se sigue con la elección de los campos comunes entre las bases, para así poder agrupar las palabras clave en el proceso investigador. En el mismo sentido, se continúa con la limpieza de la información 
resultante de las instituciones, los autores, los países y las palabras clave, ya que por falta de normalización de los metadatos en las publicaciones existe duplicación de los campos nombrados.

De igual manera, la escogencia de la base de datos wos y el periodo consultado se establecieron, en primer lugar, por el proyecto de investigación del cual se deriva presente artículo y porque la base de datos wos tiene integradas varias categorías que forman parte de su colección general. Dentro de estas categorías está ¿Scielo Citación Index, que incluye literatura latinoamericana y los documentos de artículo incluyen búsquedas de la categoría que se indagó (Narin, 1976). Asimismo, trabaja una ventana de tiempo por la obsolescencia en la literatura relaciona con el tema (Aguilera y Aguirre, 2018; Bollen et al., 2006).

\section{Resultados}

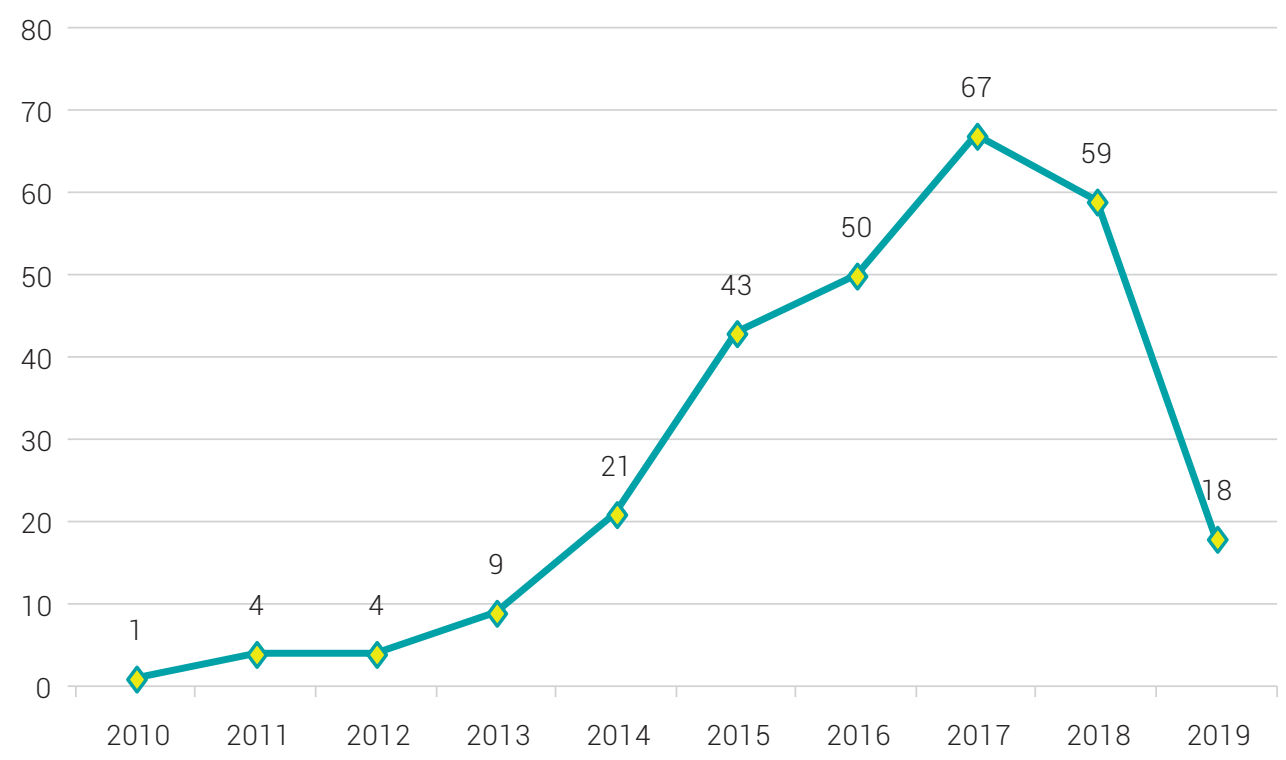

Figura 1. Documentos por año

Fuente: elaboración propia, a partir de datos de wos. 


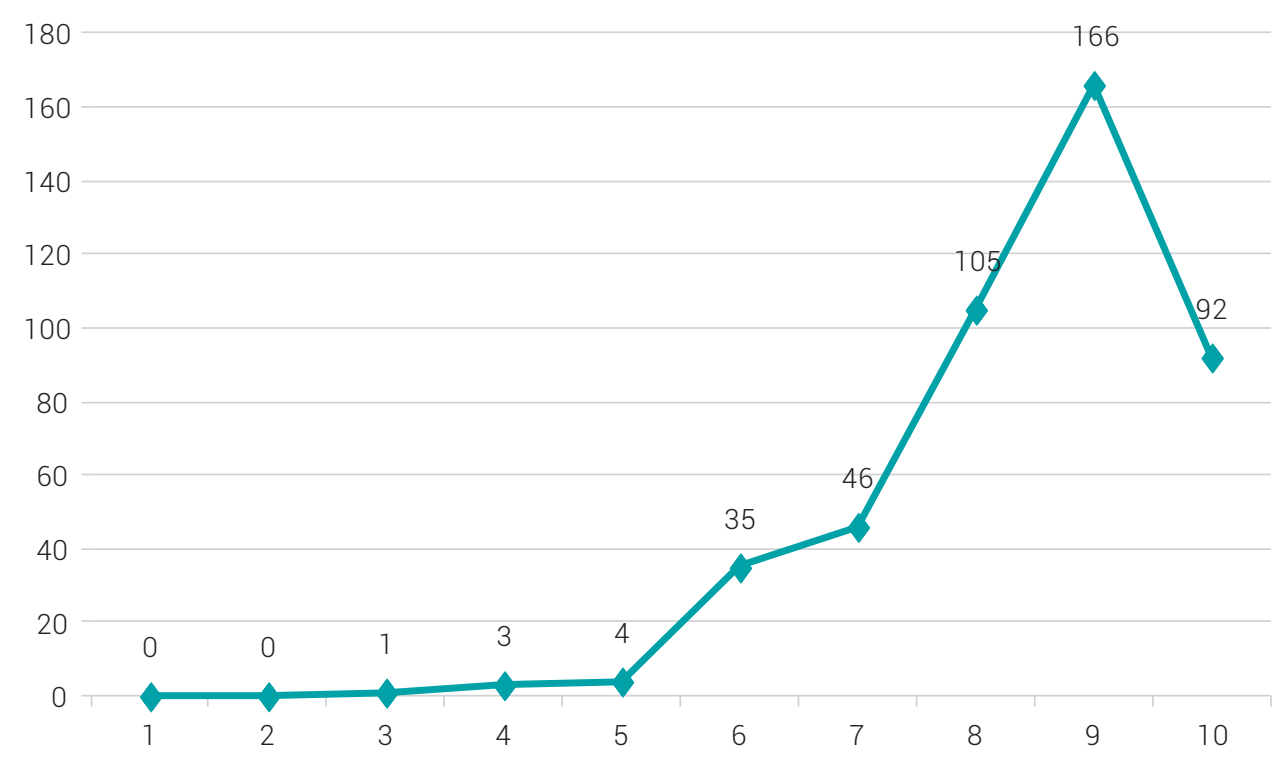

Figura 2. Citas por año

Fuente: elaboración propia, a partir de datos de wos.

Las figuras 1 y 2 identifican respectivamente los documentos por año y las citas. En la primera, el grueso se visualiza desde 2014 hasta 2018, con 240 documentos, mientras que en el periodo 2010-2013, solo hubo 18 documentos; no obstante, se presentó un crecimiento desde 2014, pero de nuevo se registró un decrecimiento para 2019. Esto último puede ser por los tiempos de publicación de las revistas de estos cuartiles, que cuentan con 12-18 meses para cumplir todos los procesos editoriales (Rivas, 2017). La figura 2 se relaciona con la producción de documentos de 2014 en adelante. En efecto, la literatura especializada establece que a medida que pasa el tiempo los artículos van teniendo mayor visualización después de ser publicados, ya sea por contenido, por la aplicación de estos o por la imagen académica de los autores o entidades (Miró y Burbano, 2013). 


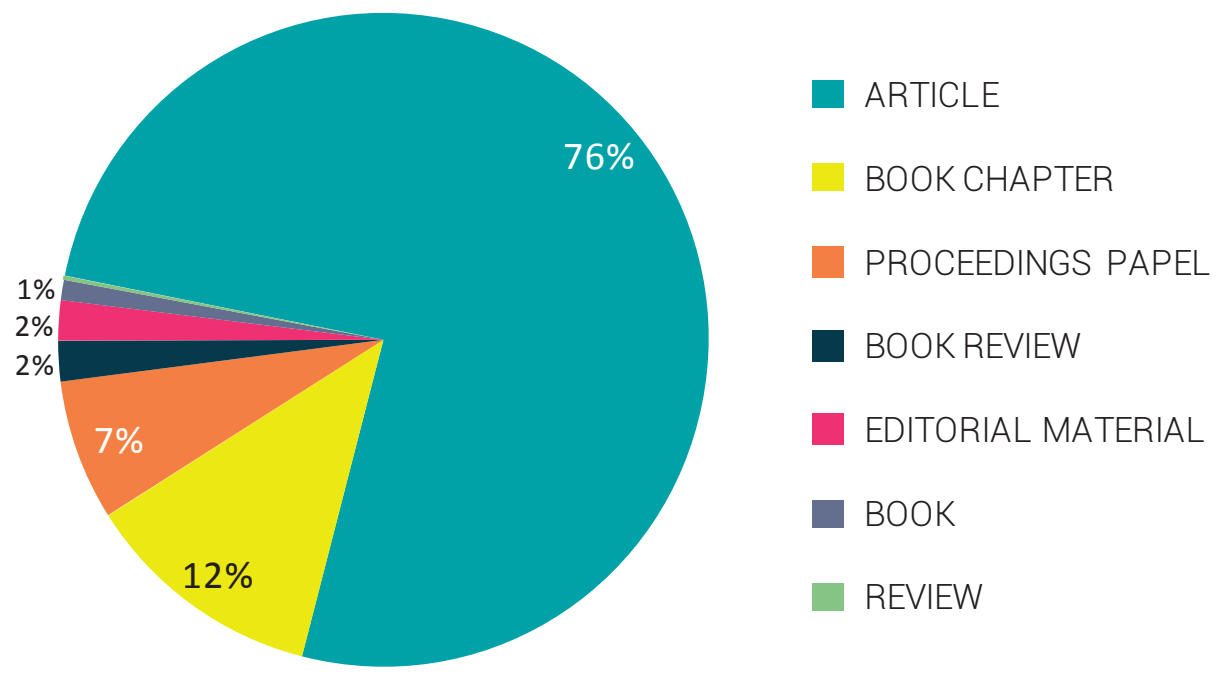

Figura 3. Tipos de documento

Fuente: elaboración propia, a partir de datos de wos

La figura 3 representa el tipo de documentos. En efecto, se evidencia una gran diferencia de producción entre artículos (un 76 \%) y el resto de los documentos. Esto puede sugerir que los primeros tienen más aceptación según las características de estos, como su nivel de impacto. Sin olvidar, el hecho de que sea más fácil de visualizar un artículo en términos nominales genera menores costos para producirlo (TorresSalinas y Cabezas-Clavijo, 2013).

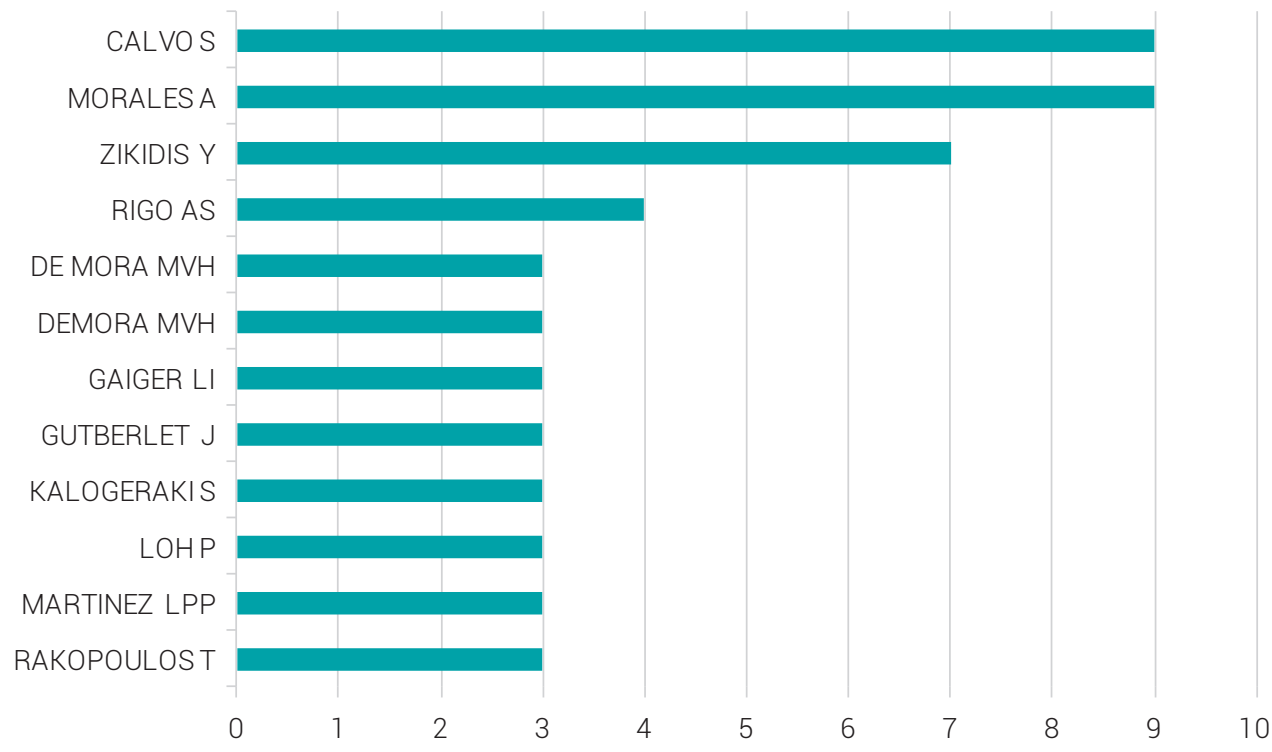

Figura 4. Documentos por autor

Fuente : elaboración propia, a partir de datos de wos. 


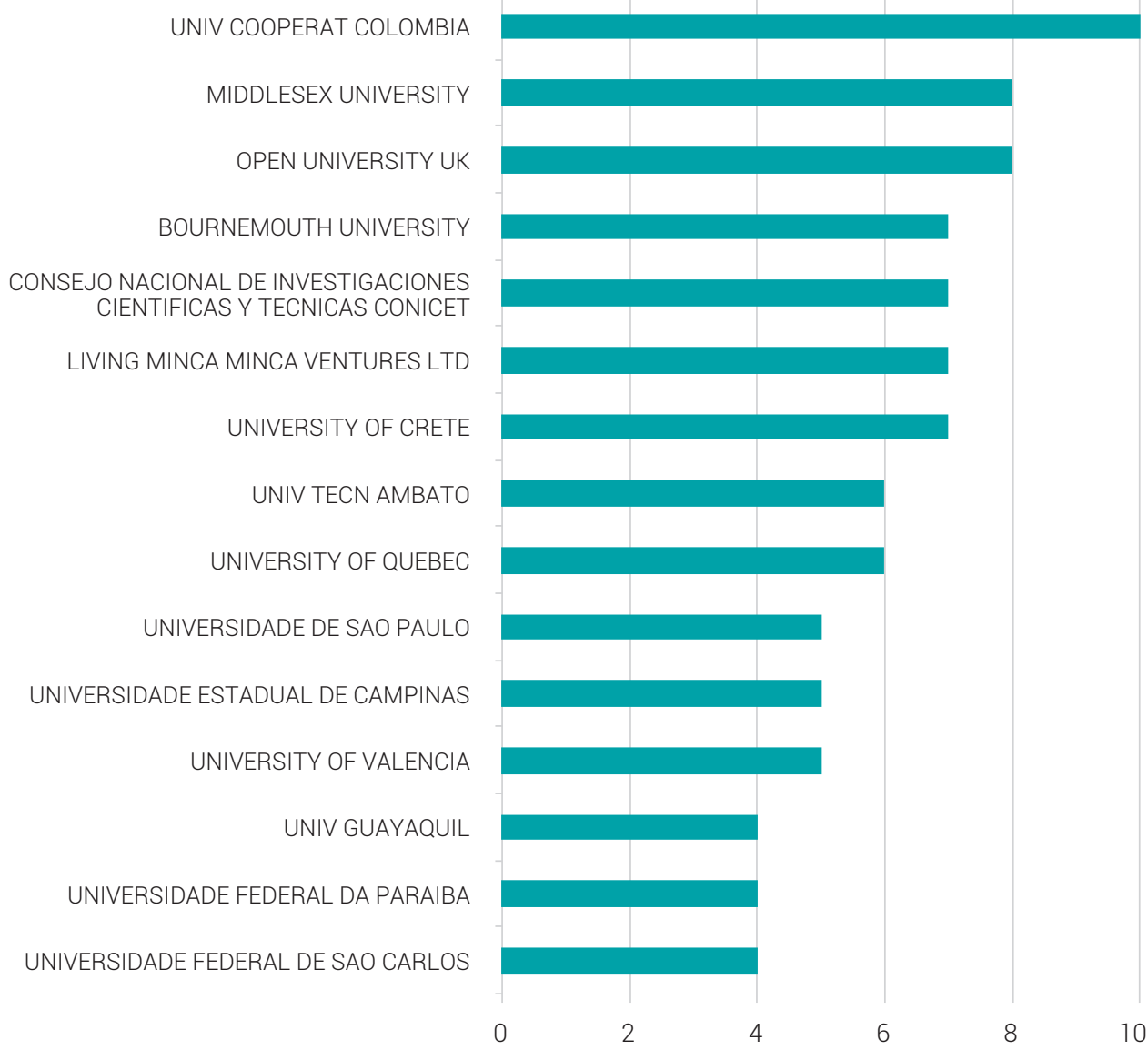

Figura 5. Documentos por organización

Fuente : Elaboración propia, a partir de datos de wos.

Las figuras 4 y 5 muestran los documentos por autor y por organización. Para la primera, hay dos autores representativos: Calvo y Morales, con nueve documentos producidos cada uno en la ventana de observación. Sin embargo, estos mismos autores no necesariamente representan a las organizaciones con mayor producción, es decir, a Universidad Cooperativa de Colombia y la a Universidad Midolesex del Reino Unido; la primera tiene diez documentos y la segunda, ocho. Esto puede sugerir que existen asimetrías entre el tridente empresa-estado-universidad, o que al ser la economía solidaria un discurso disidente hay pocas organizaciones y autores que se sintonicen con estos temas (Garfield, 1955; Dansocial , 2005). 


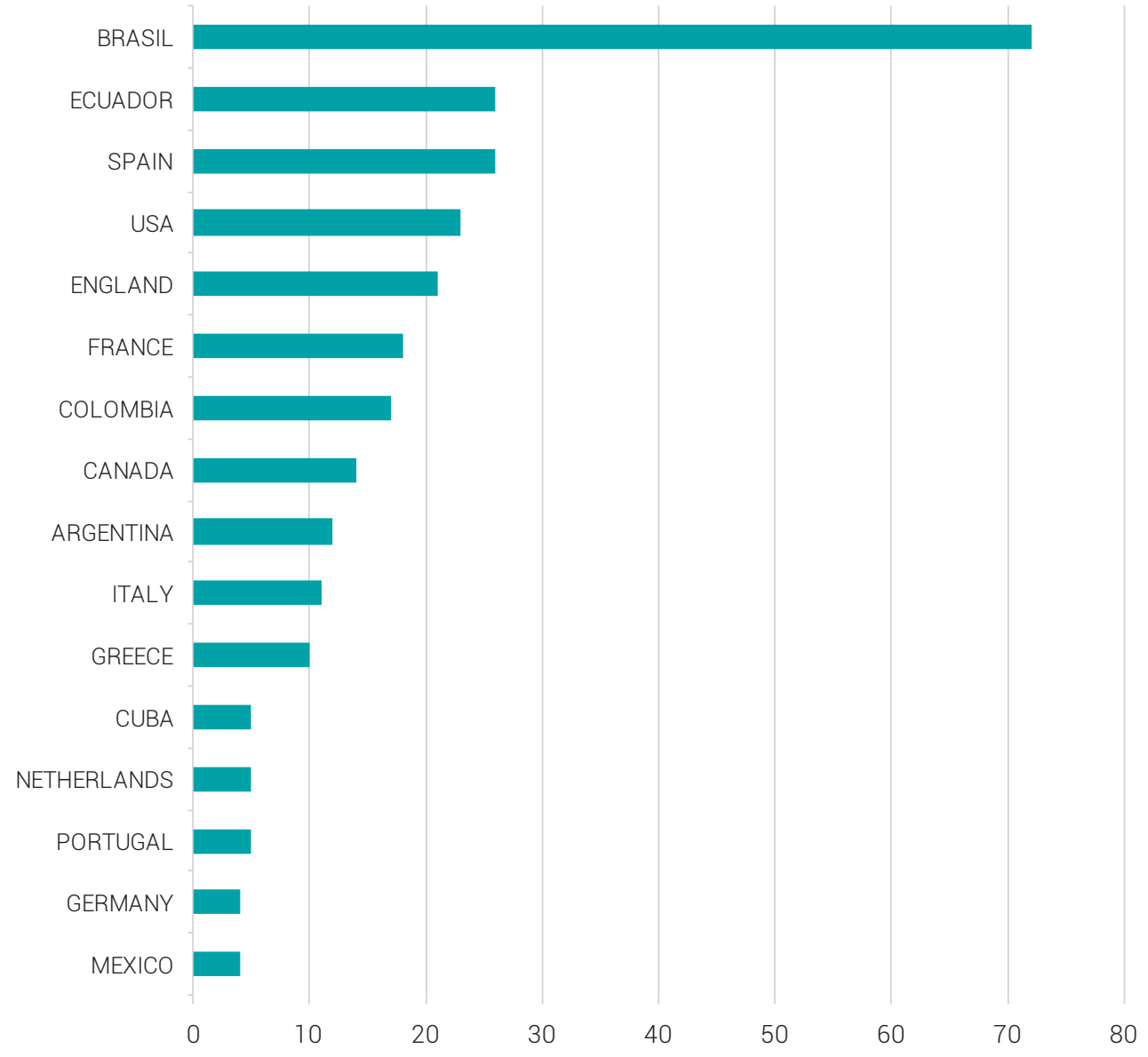

Figura 6. Registros por país

Fuente: elaboración propia, a partir de datos de wos.

Países como Brasil y Ecuador son los que tienen mayor producción (figura 6). En efecto, los desarrollos socioeconómicos y socioambientales de estas economías necesitan otras formas de entender y dinamizar las relaciones del aparato productivo con la sociedad y podrían explicar esta ventaja frente a España y Estados Unidos, que ocupan los puestos 3 y 4, respectivamente (Ministerio de Economía, 2016; Bardomiano, 2014). 


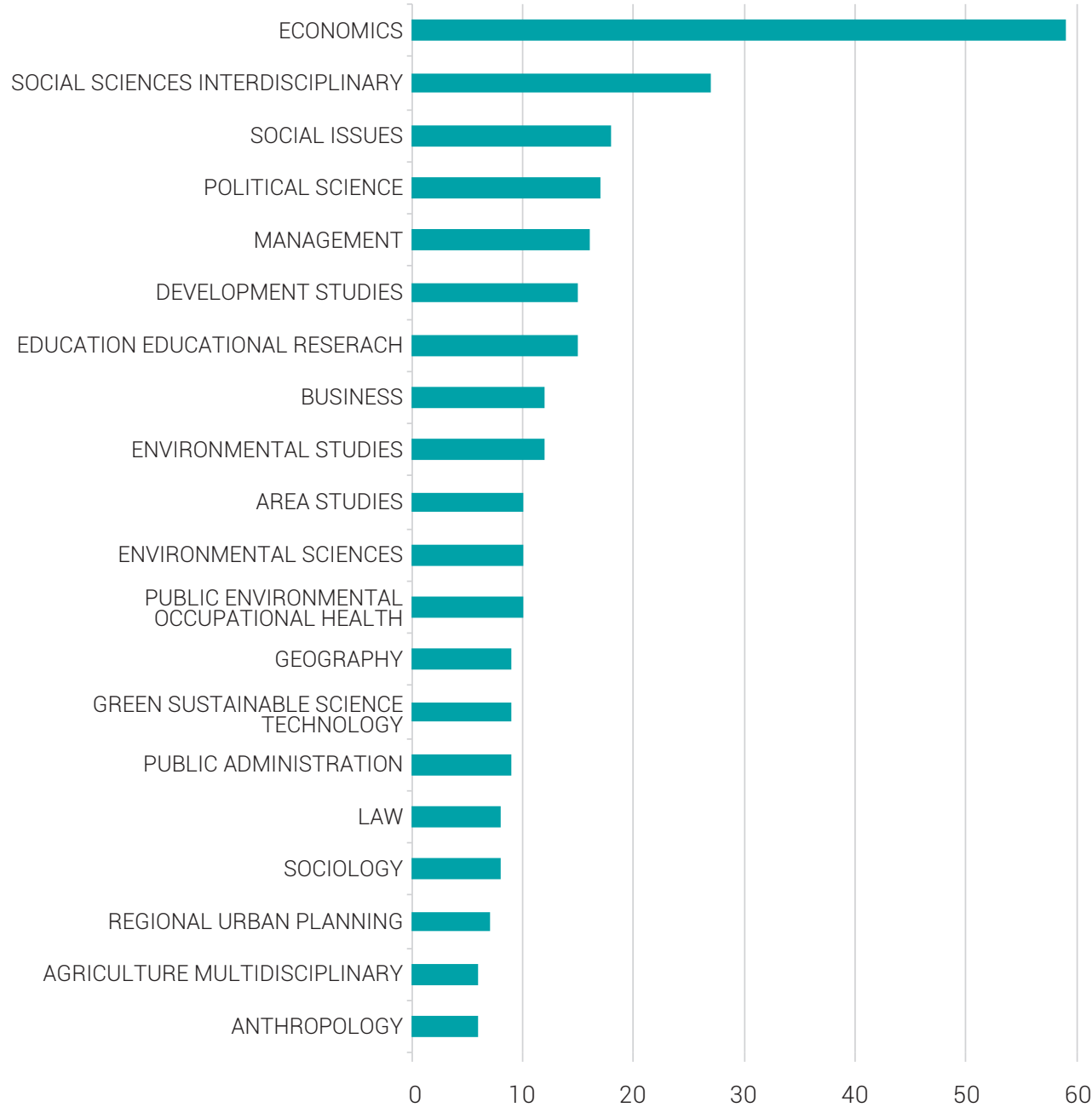

Figura 7. Documentos por área del conocimiento

Fuente: elaboración propia, a partir de datos de wos.

El área desde donde se desprende la economía solidaria es la ciencia económica, y es precisamente el área con mayor dinamismo pues registra 60 documentos (figura 7). Es importante mencionar acá que la economía solidaria es un discurso heterodoxo de la economía, lo cual se identifica en el segundo reglón de la figura 7 , que corresponde el área de ciencias sociales interdisciplinarias, en la cual se observan 25 documentos. Esto evidencia el enfoque interdisciplinario de la economía solidaria, como muy bien lo ha demostrado la literatura especializada (Barbosa et al, 2020; Rúa Castañeda et al., 2016; Zabala et al., 2018). 
Tabla 2. Documentos más citados

\begin{tabular}{|c|c|c|c|c|c|}
\hline Documento & Autores & $\begin{array}{l}\text { Título de la } \\
\text { fuente }\end{array}$ & $\begin{array}{c}\text { Año de } \\
\text { publicación }\end{array}$ & $\begin{array}{l}\text { Total de } \\
\text { citas }\end{array}$ & $\begin{array}{c}\text { Promedio } \\
\text { por año }\end{array}$ \\
\hline $\begin{array}{l}\text { Solutions to the crisis? The Green New } \\
\text { Deal, Degrowth, and the Solidarity } \\
\text { Economy: Alternatives to the capitalist } \\
\text { growth economy from an ecofeminist } \\
\text { economics perspective }\end{array}$ & $\begin{array}{l}\text { Bauhardt, } \\
\text { Christine }\end{array}$ & $\begin{array}{l}\text { ECOLOGICAL } \\
\text { ECONOMICS }\end{array}$ & 2014 & 43 & 7,17 \\
\hline $\begin{array}{l}\text { The spatiality of counter-austerity poli- } \\
\text { tics in Athens, Greece: Emergent urban } \\
\text { solidarity spaces' }\end{array}$ & $\begin{array}{l}\text { Arampatzi, } \\
\text { Athina }\end{array}$ & URBAN STUDIES & 2017 & 28 & 9,33 \\
\hline $\begin{array}{l}\text { Cooperative urban mining in Brazil: Co- } \\
\text { llective practices in selective household } \\
\text { waste collection and recycling }\end{array}$ & Gutberlet, J. & $\begin{array}{l}\text { WASTE MANAGE- } \\
\text { MENT }\end{array}$ & 2015 & 27 & 5,4 \\
\hline $\begin{array}{l}\text { Coming Full Circle Why Social and } \\
\text { Institutional Dimensions Matter for the } \\
\text { Circular Economy }\end{array}$ & $\begin{array}{l}\text { Moreau, Vin- } \\
\text { cent; Sahakian, } \\
\text { Marlyne; van } \\
\text { Griethuysen, } \\
\text { Pascal; Vuille, } \\
\text { Francois }\end{array}$ & $\begin{array}{l}\text { JOURNAL OF } \\
\text { INDUSTRIAL } \\
\text { ECOLOGY }\end{array}$ & 2017 & 26 & 8,67 \\
\hline $\begin{array}{l}\text { Resonance of Solidarity: Meanings of a } \\
\text { Local Concept in Antiausterity Greece }\end{array}$ & $\begin{array}{l}\text { Rakopoulos, } \\
\text { Theodoros }\end{array}$ & $\begin{array}{l}\text { JOURNAL OF } \\
\text { MODERN GREEK } \\
\text { STUDIES }\end{array}$ & 2014 & 19 & 3,17 \\
\hline $\begin{array}{l}\text { The power of numbers in gender } \\
\text { dynamics: illustrations from community } \\
\text { forestry groups }\end{array}$ & Agarwal, Bina & $\begin{array}{l}\text { JOURNAL OF PEA- } \\
\text { SANT STUDIES }\end{array}$ & 2015 & 16 & 3,2 \\
\hline $\begin{array}{l}\text { ALTERNATIVE FORMS OF RESILIENCE A } \\
\text { typology of approaches for the study } \\
\text { of Citizen Collective Responses in Hard } \\
\text { Economic Times }\end{array}$ & $\begin{array}{l}\text { Kousis, Maria; } \\
\text { Paschou, Maria }\end{array}$ & $\begin{array}{l}\text { PARTECIPAZIONE } \\
\text { E CONFLITTO }\end{array}$ & 2017 & 15 & 5 \\
\hline $\begin{array}{l}\text { Social Enterprise, Capabilities and } \\
\text { Development Paradigms: Lessons from } \\
\text { Ecuador }\end{array}$ & $\begin{array}{l}\text { Scarlato, } \\
\text { Margherita }\end{array}$ & $\begin{array}{l}\text { JOURNAL OF } \\
\text { DEVELOPMENT } \\
\text { STUDIES }\end{array}$ & 2013 & 13 & 1,86 \\
\hline $\begin{array}{l}\text { ALTERNATIVE FORMS OF RESILIENCE } \\
\text { CONFRONTING HARD ECONOMIC } \\
\text { TIMES: A South European Perspective }\end{array}$ & Kousis, Maria & $\begin{array}{l}\text { PARTECIPAZIONE } \\
\text { E CONFLITTO }\end{array}$ & 2017 & 12 & 4 \\
\hline $\begin{array}{l}\text { Solidarity economy and community } \\
\text { development: emerging cases in three } \\
\text { Massachusetts cities }\end{array}$ & $\begin{array}{l}\text { Loh, Penn; } \\
\text { Shear, Boone }\end{array}$ & $\begin{array}{l}\text { COMMUNITY } \\
\text { DEVELOPMENT }\end{array}$ & 2015 & 12 & 2,4 \\
\hline
\end{tabular}

Fuente: elaboración propia, a partir de datos de wos.

La tabla 2 ratifica que la ciencia se escribe en el idioma inglés. Todos los documentos identificados están en este idioma y esto concuerda con las relaciones geopolíticas, como las exigencias de las revistas en promedio de alto impacto (Davidson y Delbridge, 2011; Björk y Solomon, 2012). Además, los documentos identificados también representan la amplitud de temas y los aportes de la economía solidaria, dadas sus características heterodoxas (Razeto, 1992). 


\section{Discusión}

El concepto de economía solidaria está constituido por experiencias basadas en principios de solidaridad y apoyo mutuo, y que tienen como base la horizontalidad entre sus integrantes (Osorio, 2017). Además, busca fortalecerse por medio de la creación de redes de cooperación, y de esta manera construir una posibilidad real de creación de relaciones equitativas y justas entre sus miembros, y pone en el centro al ser humano y su entorno medio ambiental (Departamento Administrativo Nacional de la Economía Solidaria, 2005), a diferencia del discurso hegemónico de la economía ortodoxa (Rubio Rodríguez et al., 2019; Losada et al., 2019).

Por otra parte, la economía solidaria proyecta el desarrollo como un proceso en el que los aportantes de factores los ejecuten con independencia en contextos asociativos empresariales. De igual forma, contempla un proyecto de mercado democrático y de mercado solidario con el fin de modificar la manera de aplicación del desarrollo con una concepción diferente de este (Caillé, 2009; Gómez Rodríguez, 2020).

En la academia, la economía solidaria comienza a ser reconocida como una modalidad de organización colectiva con características distintivas. Amaro (2009) indica que la economías solidaria se caracteriza por tres aspectos: 1) policéntrico: surge de manera autónoma en los territorios compartiendo líneas de base; 2) polisémico: porque adquiere diferentes significados dependiendo del entorno en el que se aplique, y 3) poliexpresión: se relaciona con el tipo de prácticas. De igual forma, el autor plantea tres enfoques de la economía solidaria estudiados en la academia: 1) la versión francófona, que propone la idea de una economía plural que compendia Estado, mercado y sociedad civil. Esta versión es la más desarrollada teóricamente; 2) la versión latinoamericana, más popular y comunitaria, que valora también el carácter de proyecto cultural y ambiental, que se puede identificar en Colombia con los trabajos de la Universidad Cooperativa de Colombia, la Universidad de La Salle, Unicossol y la Unidad Administrativa Especial de Organizaciones Solidarias, en concordancia con el presente escrito, y 3) la propuesta de Macaronesia (zona compuesta por cinco archipiélagos del Atlántico Norte: Azores, Canarias, Cabo Verde, Madeira e Islas Salvajes), donde la transformación integral y una visión ecocéntrica constituyen sus puntos más importantes. A partir de lo anterior se puede decir que existen dos enfoques fundamentales en el contexto de la economía solidaria: la orientación europea con importantes relaciones en Quebec (Canadá) y el enfoque latinoamericano, en especial Chile, Argentina y Brasil (Chaves Ávila, 2006).

La economía solidaria tiene como antecedente un concepto más antiguo denominado economía social, el cual está relacionada con experiencias formales de mutualismo y cooperativismo. Existe un debate académico que busca concatenar 
los dos conceptos en uno solo denominado economía social y solidaria (Estivill, 2012; Laville, 2004), con objeto de dejar atrás una división existente entre los dos aspectos en los que se le critica a la economía social la pérdida de orientación política transformadora y en la que la economía solidaria sí es vista como una alternativa real al capitalismo (Vargas y Sánchez, 2020). Esto, sin olvidar que hay pocas universidades en el mundo que tienen como fortaleza robustecer estos procesos desde los postulados de la economía solidaria (Gómez, 2009; Pardo Martínez et al., 2006).

\section{Conclusiones}

La economía solidaria constituye un concepto que permite pensar y actuar en métodos alternativos de generación de actividades productivas de empleo y mejora de la calidad de la vida de la población. Precisamente, el presente artículo representa un esfuerzo por esquematizar de manera formal las búsquedas académicas en bases de datos especializadas del concepto de economía solidaria, con el propósito de contar con información fiable que permita enfocar los intereses investigativos.

La ventana de observación entre el periodo 2010-2019 evidencia y ratifica cómo a medida que transcurre el tiempo hay un repunte en las publicaciones sobre economía solidaria hasta 2017. Asimismo, se refleja de qué manera partir de ese año hubo un descenso continuo desde el momento cuando se realizó el estudio bibliométrico. En el mismo sentido, la gran mayoría de los documentos identificados en la base de datos wos concluyen contundentemente que los aportes se basan en artículos científicos (76\%) y que están muy alejados de los capítulos de libro. De igual modo, existen dos autores que son los que generan tendencia: Calvo y Morales; asimismo, las organizaciones con mayores aporte son la Universidad Cooperativa de Colombia y la Bournemouth University. Para terminar, entre los países con mayor producción se encuentran Brasil y Colombia, que ocupa el séptimo puesto, lo cual ratifica la necesidad de ampliar como enriquecer el acervo científico del país frente a la economía solidaria.

\section{Referencias}

Aguilera-Padro, M., Rincón Moreno, H. y Gómez-Rodríguez, D. (2020). Bioeconomía, una alternativa de investigación en administración y afines. En M. Aguilera-Prado y H. Rincón Moreno (Eds.), Temas y métodos de investigación en negocios, administración, mercadeo y contaduría (pp. 193-225). Editorial Uniagustiniana. 
Aguilera-Prado, M. y Aguirre Garzón, C. (2018). Tendencias de la literatura en administración de empresas. En Lecciones de investigación para la competitividad empresarial (pp. 63-73). Corporación Universitaria Americana.

Álvarez Rodríguez, J. F. y López-Santamaría, M. (Comps.). (2018). Apuntes para el fomento de la asociatividad solidaria y el logro de los objetivos de desarrollo sostenible. Unidad Administrativa Especial de Organizaciones Solidarias (UAEOS); Centro de Investigación, Documentación e Información de la Economía Social, Pública y Cooperativa (CIRIEC).

Amaro, R. (2009). A Economia Solidária da Macaronésia - Um novo conceito. Revista Economía Solidária, (1), 11-30.

Barbosa, E., Merchán, O. y Gómez, D. (2020). La economía solidaria como potenciador del desarrollo rural y de la construcción de la paz en Colombia. Ponencia. En IV Seminario Internacional de Desarrollo y Territorio. Universidad de La Salle, Bogotá, Colombia.

Bardomiano, M. (2014). Ecuador: panorama y evolución económica reciente. Economía Informa, (385), 100-108.

Bastidas, O. (2015). Curso radial. Organización y funcionamiento de las organizaciones de economía solidaria con énfasis en las cooperativas. http://Downloads/!\%200Bastidas\%20Curso \%20Radial\%200rg\%20y\%20Func\%20de\%20las\%200ESol\%20Colombia\%20240815\%20 (4).pdf

Bensman, S. y Leydesdorff, L. (2009). Definition and identification of journals as bibliographic and subject entities: Librarianship versus ISI Journal Citation Reports methods and their effect on citation measures. Journal of the American Society for Information Science and Technology, 60(6), 1097-1117.

Björk B. y Solomon, D. (2012). Open access versus subscription journals: a comparison of scientific impact. BMC Medicine, 10(1), 73.

Bollen, J., Rodríguez, M. y Van de Sompel, H. (2006). Journal status. Scientometrics, 69(3), 669-687.

Caillé, A. (2009), Sobre los conceptos de economía en general y de economía solidaria en particular. En J. L. Coraggio (Org.), ¿Qué es lo económico? Materiales para un debate necesario contra el fatalismo. Ediciones CICCUS.

Castro Jiménez, L. E., Rincón Moreno, M. y Gómez Rodríguez, D. T. (2017). Educación para la salud: una mirada desde la antropología. Revista Ciencias de la Salud, 15(1), 145-163, http://dx.doi. org/10.12804/revistas.urosario.edu.co/revsalud/a.5387 
Cueto Fuentes, E. N, Arboleda Álvarez, O. L, Zabala Salazar, H. y Echeverry Correa, F. J. (2018). Una década de economía social y solidaria en Colombia: análisis de la producción investigativa y académica 2005 - 2015.Bogotá, Colombia: Corporación Universitaria Minuto de Dios - UNIMINUTO y Universidad Católica Luis Amigó.

Chaves Ávila, R. (2006). La economía social en la Unión Europea: resumen del informe elaborado para el Comité Económico y Social Europeo por el CIRIEC. Noticias de la Economía Pública Social y Cooperativa, (49), 42-55.

Dávila, R., Vargas, A., Blanco, L., Roa, E., Cáceres, L.E. y Vargas, L.A. (2018). Características de la economía solidaria colombiana. Aproximaciones a las corrientes influyentes en Colombia, CIRIEC-España. Revista de Economía Pública, Social y Cooperativa, (93), 85-113. 10.7203/ CIRIEC-E.93.10327

Davidson, A. y Delbridge, E. (2011). How to write a research paper. Paediatrics and Child Health, 22(2), 61.65.

Departamento Administrativo Nacional de la Economía solidaria. (2005). Inventario sistematizado de experiencias en educación solidaria en Colombia. Imprenta Nacional de Colombia.

Escorcia Otálora, T. A. (2008). El Análisis bibliométrico como herramienta para el seguimiento de publicaciones de publicaciones científicas, tesis y trabajo de grado. (Tesis de grado). Pontificia Universidad Javeriana, Colombia. https://www.javeriana.edu.co/biblos/tesis/ciencias/tesis209.pdf

Estivill, J. (2012). Espacios públicos y privados. Construyendo diálogos en torno a la economía solidaria. Revista Crítica de Ciencias Sociales, (84), 101-113.

Garfield E. (1955). Citation indexes for science: a new dimension in documentation through association of ideas. Science, 122(3159), 108-11.

Gepp, A., Linnenluecke, M. K., O’Neill, T. y Smith, T. (2018). Big data techniques in auditing research and practice: Current trends and future opportunities. Journal of Accounting Literature, 40, 102-115.

Gómez Rodríguez, D. T. (2020). Solidaridad en época de pandemia. Aracataca, Magdalena: cuna del premio Nobel de literatura. En J. Wilches y A. Castiblanco Roldán (Eds.), Criaturas en el encierro: reflexiones en tiempos de coronavirus (pp. 65-72). Universidad Distrital Francisco José de Caldas. 
Gómez Rodríguez, D. T., Carranza Abella, Y. y Ramos Pineda, C. A. (2017a). Revisión documental, una herramienta para el mejoramiento de las competencias de lectura y escritura en estudiantes universitarios. Chakiñan, Revista de Ciencias Sociales y Humanidades, (1), 46-56.

Gómez Rodríguez, D. T., Carranza Abella, Y. y Ramos Pineda, C. A. (2017b). Ecoturismo, turismo experiencial y cultural. Una reflexión con estudiantes de Administración de Turismo. Revista TEMAS, 3(11), 229-236.

Gómez Rodríguez, D. T., Carranza Abella, Y. y Ramos Pineda, C. A. (2017c). Aportes de negociadores internacionales para el crecimiento, la innovación, el emprendimiento y la competitividad de las empresas colombianas. Revista FACCEA, 7(2), 157-164.

Gómez, M. (2009). De la economía solidaria al desarrollo sustentable: una aproximación a través de las prácticas pedagógicas de la Universidad Cooperativa de Colombia. En Memorias del V Seminario Internacional Universidad y Ambiente de Cara Al Cambio climático. Ediciones Universidad Cooperativa de Colombia.

Laville, J. (2004). El marco conceptual de la economía solidaria. En Economía social y solidaria. Una visión europea (pp. 207-236). Altamira.

Laville, J. L. (2007). L'économie Solidaire, une perspective internationale. C. H. Sociologie.

Martorell, M., y Santos, J. (2012). Manual de historia política y social de España (1808-2011). rba.

Laville, J. L., Levesque, B. y Mendell, M. (2006). The Social Economy. Diverse approaches and practices in Europe and Canada. Quebec. Bibliothéque et Archives Canada.

Ley 454 de 1998. "Por la cual se determina el marco conceptual que regula la economía solidaria, se transforma el Departamento Administrativo Nacional de Cooperativas en el Departamento Nacional de la Economía Solidaria, se crea la Superintendencia de la Economía Solidaria, se crea el Fondo de Garantías para las Cooperativas Financieras y de Ahorro y Crédito, se dictan normas sobre la actividad financiera de las entidades de naturaleza cooperativa y se expiden otras disposiciones". Congreso de Colombia. https://www.funcionpublica.gov.co/eva/ gestornormativo/norma.php?i=3433Losada, J., Valencia, S. y Gómez, D. (2018). La gestión de talento humano en el desarrollo organizacional. CrearE, Cuadernos de Emprendimiento, 3, 62-69.

Ministerio de Economía. (2016). La economía de Brasil. Situación y perspectiva. https://www. santafe.gov.ar/index.php/web/content/download/231947/1212623/version/2/file/Brasilnoviembre.pdf 
Miró, Ò. y Burbano, P. (2013) El factor de impacto, el índice h y otros indicadores bibliométricos. Anales Sis San Navarra, 36(3), 371-377.

Monsalve, A. (2018). Pedagogías de la acción solidaria para construir desarrollo sostenible. Unidad Administrativa Especial de Organizaciones Solidarias (UAEOS) y Asociación Colombiana de Cooperativas (ASCOOP).

Narin, F. (1976). Evaluative bibliometrics: The use of publication and citation analysis in the evaluation of scientific activity. Cherry Hill, NJ: Computer Horizons. Organización para la Cooperación y el Desarrollo Económicos. https://www.researchgate.net/publication/269992229_Evaluative_ Bibliometrics_The_Use_of_Publication_and_Citation_Analysis_in_the_Evaluation_of_ Scientific_Activity

Nunes Alves, J., Flaviano, V., Klein, L. L., Leodir Löbler, M. y Diniz Pereira, B. A. (2016). A Economia Solidária no Centro das Discussões: um trabalho bibliométrico de estudos brasileiros. Cadernos EBAPE.BR,14(2), 243-257. http://dx.doi.org/10.1590/1679-395120257

Osorio, M. (2017). Modos de vida vivibles: economía(s) solidaria(s) y sostenibilidad de la vida. Barcelona. Universidad Autónoma de Barcelona.

Pardo Martínez, L. P. (Dir.) (2006). Estado del Arte de la economía solidaria en Bogotá. Ediciones Universidad Cooperativa de Colombia.

Pérez, P., y Uribe, V. (2016). Reflexiones para conceptualizar territorio solidario. Revista ÁGORA.,16 (2), 533- 546.

Pérez, N. (2002). La bibliografía, bibliometría y las ciencias afines. ACIMED, 10(3), 1-2.

Razeto, L. (1992). Fundamentos de la teoría económica comprensiva. Pet.

Razeto, L. (1997). Los caminos de la economía de solidaridad. Lumen Humanitas.

Razeto, L. (2014). Economía de la solidaridad. http://www.uvirtual.net/spuv/catedra-latinoamericana-de-economia-solidaria

Rivas, F. (2017). Publicar en revistas de impacto. En AEPap (Ed.), Curso de actualización pediatría (pp. 409-418). Lúa Ediciones.

Rúa Castañeda, S., Monroy Flores, V. E., Peñuela Lizcano, J. D., Pérez Villa, P. E., Calderón Ibáñez, A. del C., Arenas Sepúlveda, C. C., y Jiménez Patiño, H. D. (2016). Integración económica solidaridad en territorio. Aportes a la construcción de modelos y metodologías. Unidad Administrativa Especial de Organizaciones Solidarias (UAEOS); Universidad Cooperativa de Colombia. 
Rubio Rodríguez, G. A., Téllez Bedoya, C. A. y Gómez Rodríguez, D. T. (2019). Análisis de los factores que componen un sistema de gestión empresarial: estudio de caso para la Dirección de Vivienda. Revista Nacional de Administración, 10(2), 47-60.

Solano López, E., Castellanos Quintero, S., López Rodríguez del Rey, M. y Hernández Fernández, J. (2009). La bibliometría: una herramienta eficaz para evaluar la actividad científica postgraduada. MediSur, 7(4), 59-62.

Torres-Salinas, D. y Cabezas-Clavijo, Á. (2013). Cómo publicar en revistas científicas de impacto: consejos y reglas sobre publicación científica. EC Work Pap, (31), 1-16.

Unidad Administrativa Especial de Organizaciones Solidarias (UAEOS). (2019a).. La Unidad Administrativa y el sector solidario en el nuevo PND. https://www.orgsolidarias.gov.co/ Prensa-noticias-la-unidad-administrativa-y-el-sector-solidario-en-el-nuevo-PND

Unidad Administrativa Especial de Organizaciones Solidarias (UAEOS). (2019b). Plan Nacional de Desarrollo Pacto por Colombia, Pacto por la equidad 2018-2022. Ley 1955 de 2019. https:// www.orgsolidarias.gov.co/sites/default/files/archivos/PND\%20documento\%20UAEOS.PDF

Vargas-Prieto, A. y Sánchez-Álvarez, C. (2020). Análisis de evolución de la asistencia técnica y el fomento de cooperativas rurales en Colombia. Cooperativismo \& Desarrollo, 27(116)1-22. doi: https://doi.org/10.16925/2382-4220.2020.01.03

Vainstok, A (1985). Economía social. Nuevo País.

Vélez Tamayo, J. M. (2014). Construcción del concepto de economía solidaria: una mirada a la teoría económica comprensiva. Cooperativismo \& Desarrollo, 22(105), 17-29. http://dx.doi. org/10.16925/co.v22i105.690

Zabala, H., Arboleda, O. y Cueto, E. (2018). Aproximaciones a una antropología de la cooperación. Science of Human Action, 3(1), 16-33. 